\title{
ENFERMAGEM NA SAÚDE DA CRIANÇA: ESTUDO BIBLIOGRÁFICO ACERCA DA AVALIAÇÃO NUTRICIONALa
}

\author{
Nursing in child's health: bibliography study on assessment \\ nutritional
La enfermería en la salud del niño: estudio bibliográfico sobre la evaluación nutricional

Flávia Paula Magalhães Monteiro'

Joselany Áfio Caetano²

Thelma Leite de Araujo ${ }^{3}$

\section{RESUMO}

0 objetivo deste estudo foi analisar a produção científica acerca da atuação do enfermeiro na saúde nutricional da criança. Estudo bibliográfico realizado entre abril e maio de 2008, nas bases de dados online, com descritores: avaliação nutricional, antropometria, crescimento, criança. Critérios de inclusão: saúde infantil na avaliação nutricional, enfermeiro/autor e artigos publicados entre 2000 e 2007. Encontraram-se 15 artigos nos seguintes anos: 2000 (1), 2001 (1), 2002 (2), 2003 (4), 2005 (1), 2006 (3) e 2007 (3). Periódicos inscritos nas áreas: enfermagem (1), nutrição (1), saúde pública (3) e médica (1). Tipo de estudo: epidemiológicos (1), descritivos (5), transversais (7), longitudinal (1), estudo de caso (1), revisão de literatura (1) e relato de experiência (1). Unidades temáticas: avaliação nutricional mediante acompanhamento do crescimento e desenvolvimento infantil; fatores determinantes da nutrição infantil e avaliação nutricional como cuidado de enfermagem. Ressalta-se que o enfermeiro tem buscado fundamentação teórica e prática para cuidar da criança com déficit nutricional.

Palavras-chave: Avaliação Nutricional. Criança. Enfermagem.

\begin{abstract}
To examine the scientific production about the role of nurses in the nutritional health of child. Bibliographic study, held between April and May 2008 in on line databases, with descriptors: nutritional assessment, anthropometry, growth, child. Criteria for inclusion: child health in nutrition assessment, nurse / author and articles published between 2000 and 2007. Meeting 15 articles in the years: 2000 (1), 2001 (1), 2002 (2), 2003 (4), 2005 (1), 2006 (3) e 2007 (3). Regular subscribers in the areas: nursing (1), nutrition (1), health (3) and medical (1). Type of study: epidemiological (1), descriptive (5), Cross (7), longitudinal (1), the case study (1), review of literature (1) and report of experience (1). Thematic units: nutritional assessment by the monitoring of growth and child development by nurses; determinants of children's nutrition and nutritional assessment and nursing care. It is emphasized that the nurse has sought theoretical and practical reasons to take care of children with nutritional deficiency.
\end{abstract}

Keywords: Nutrition assessment. Child. Nursing.

\section{Resumen}

Analizar la producción científica que existe sobre el papel que desempeña el enfermero en la salud nutricional del niño. Estudio bibliográfico desarrollado entre abril y mayo/2008 usando bases de datos en línea, a través de los siguientes descriptores: evaluación nutricional, antropometría, crecimiento, niño. Se aplicaron los siguientes criterios de inclusión: salud infantil en la evaluación nutricional, enfermero/ autor y artículos publicados entre 2000 y 2007 . Se encontraron 15 artículos en los años: 2000 (1), 2001 (1), 2002 (2), 2003 (4), 2005 (1), 2006 (3) e 2007 (3). Periódicos inscriptos en las áreas: enfermería (1), nutrición (1), salud pública (3) y médica (1). Tipo de estudio: epidemiológicos (1), descriptivos (5), transversales (7), longitudinales (1), estudio de caso (1), revisión de literatura (1) y relato de experiencia (1). Unidades temáticas: evaluación nutricional mediante acompañamiento del crecimiento y desarrollo infantil; factores determinantes de la nutrición infantil y evaluación nutricional como cuidado de la enfermería. Descata el hecho que el enfermero ha buscado desarrollar una fundamentación teórico/práctica para cuidar del niño con déficit nutricional.

Palabras clave: Evaluación nutricional. Niño. Enfermería.

\footnotetext{
'Enfermeira. Aluna do Curso de Mestrado em Enfermagem - UFC. Bolsista do CNPq vinculada ao Projeto Cuidado em Saúde Cardiovascular/CNPq processo n: 551337/2008-7. Brasil. E-mail: flaviapmm@yahoo.com.br, ${ }^{2}$ Doutora em Enfermagem. Professora da disciplina Metodologia científica do Programa de Pós-Graduação em Enfermagem da Universidade Federal do Ceará (UFC). Brasil. E-mail: joselany@ufc.br, ${ }^{3}$ Doutora em Enfermagem. Professora Associada do Departamento de Enfermagem da Universidade Federal do Ceará (UFC). Brasil. E-mail: thelma@ufc.br
} 


\section{INTRODUÇÃO}

Em suas diferentes abordagens de cuidado, o enfermeiro exerce papel fundamental na assistência à criança, iniciado na fase da vida uterina, durante as consultas de enfermagem no pré-natal e por meio do exame físico materno, quando é possível identificar anormalidades e viabilizar condutas com vistas ao crescimento e desenvolvimento do concepto. Tais condutas, em caso de alterações, podem salvar a vida de ambos, mãe e filho.

Em continuidade a esse cuidado, outras atividades de enfermagem podem ser implementadas ao longo dos primeiros meses de vida da criança. Em relação às consultas de acompanhamento do crescimento e desenvolvimento infantil, de modo geral, são feitas mensurações dos índices antropométricos, que incluem peso, estatura, perímetros cefálico, torácico e abdominal, pregas cutâneas tríceps e subescapular. A avaliação deve ser feita periodicamente pelo enfermeiro na tentativa de investigar alterações provenientes de doenças que desencadeiam anormalidades na estrutura morfológica e estrutural do corpo infantil.

Como parte das consultas de acompanhamento do crescimento e desenvolvimento infantil realizadas pelo enfermeiro na atenção básica de saúde, inclui-se a avaliação nutricional, cujo objetivo é verificar o crescimento e as proporções corporais em um indivíduo ou em uma comunidade, no intuito de estabelecer atitudes de intervenção.

Como recomendado, é de fundamental importância a padronização da avaliação a ser utilizada para cada faixa etária, uniformizando assim os critérios empregados pela equipe de saúde. ${ }^{1}$

A partir dessa consulta, o enfermeiro pode identificar prováveis respostas humanas que culminam na elaboração de diagnósticos de enfermagem e, precocemente, estabelecer condutas preventivas ou intervencionistas destinadas a possibilitar o processo de cuidar da criança. Além disso, as informações levantadas sobre o peso e outros índices antropométricos nas consultas de enfermagem facilitam 0 diálogo e o aconselhamento da mãe ou responsáveis sobre os cuidados com alimentação, adequação do peso para a idade da criança, sinais e sintomas de desnutrição.

Para o enfermeiro, esta avaliação se constitui na anamnese alimentar, no exame físico direcionado à identificação de índices antropométricos e na análise de métodos bioquímicos. Tal avaliação é feita com a finalidade de acompanhar o crescimento e o desenvolvimento infantil e, ao mesmo tempo, estabelecer a monitoração constante das variáveis antropométricas, consideradas significativos indicadores de saúde. Inclui, ainda, identificar os fatores de risco, tais como: baixo peso ao nascer, desmame precoce, existência de doenças perinatais, histórico de desnutrição materna. ${ }^{2}$

Como membro da equipe multiprofissional, o enfermeiro deve atuar na prevenção, deteç̧ão e tratamento da desnutrição, conforme a legislação vigente, sobretudo a Portaria 272 e a Resolução 63 do Ministério da Saúde. ${ }^{3}$

No entanto, ainda existem dúvidas sobre qual a extensão e profundidade da avaliação nutricional de enfermagem. Nem sempre há consenso sobre seus componentes, nem sobre quais dados são importantes e de quais instrumentos propedêuticos o enfermeiro deve lançar mão para uma avaliação nutricional geradora de dados relevantes para o cuidado de enfermagem.

$\mathrm{Na}$ tentativa de responder a esses questionamentos, este estudo pretende analisar a produção científica de enfermagem no que diz respeito à atuação do enfermeiro na saúde da criança com ênfase na avaliação nutricional, com vistas a estabelecer um panorama atual acerca da temática em discussão, bem como trazer subsídios à organização da assistência de enfermagem a esse segmento populacional.

\section{METODOLOGIA}

Estudo de caráter descritivo, realizado por meio de uma pesquisa bibliográfica durante os meses de abril e maio de 2008. 0 estudo bibliográfico compreende etapas, como a formulação do problema, a escolha do tema a ser investigado, a elaboração do plano de trabalho, a formulação dos objetivos, identificação, localização e obtenção de fontes que sejam capazes de fornecer os dados adequados à pesquisa desejada, leitura do material obtido, análise e interpretação lógica dos dados e redação final do texto. ${ }^{4}$

Inicialmente, foram adotados como fontes os artigos publicados em periódicos nas línguas portuguesa, inglesa e espanhola, indexados nas bases de dados informatizadas, tais como LILACS (Literatura Latino-Americana e do Caribe em Ciências da Saúde), MedLine, SCIELO e BDENF (Base de Dados de Enfermagem - Bireme). Para este fim, localizaramse os descritores como indexadores da busca, registrados na Bireme: avaliação nutricional, antropometria, crescimento e criança, os quais foram submetidos a cruzamentos entre si na tentativa de se encontrar a produção científica correspondente. Os artigos pesquisados contemplaram os seguintes critérios de inclusão: temas relacionados à saúde da criança com ênfase na avaliação nutricional, a inserção do enfermeiro como autor e artigos publicados no período de 2000 a 2007. Considerouse artigo a produção científica cuja meta foi estabelecer descobertas em arquivos indexados ou de renome nacional e internacional..$^{5}$ 
Nas bases de dados pesquisadas, foi encontrado um total de 612 publicações. A caracterização dos artigos foi feita mediante sua leitura na íntegra, buscando na sua totalidade os descritores. Foram eliminados artigos que se repetiam nas bases de dados. Desta forma, foram selecionadas 15 publicações que atenderam aos critérios de inclusão. Depois disso, procedeu-se à leitura exaustiva dos artigos na íntegra, na tentativa de se obter informações centrais, que foram organizadas em unidades temáticas para facilitar a análise e a compreensão do assunto, destacando ainda os seguintes aspectos: ano de publicação, área de conhecimento do periódico, tipo de estudo, autorias, temas de estudo e abrangência.

Por se tratar de um estudo bibliográfico, não foi necessário submeter o projeto à avaliação de Comitê de Ética em Pesquisa com Seres Humanos, conforme determina a Resolução 196/96 do Ministério da Saúde. ${ }^{6}$

\section{RESULTADOS E DISCUSSÃO}

Os 15 artigos selecionados foram publicados, na sua maioria, em periódicos datados do ano de 2003 (4 artigos), seguido dos anos de 2006 (3 artigos), 2007 (3 artigos), 2002 (2 artigos) e dos anos 2000, 2001 e 2005, com apenas uma publicação cada. Esses periódicos são apresentados nas bases de dados como inscritos nas áreas de enfermagem (1), nutrição (1), saúde pública (3) e médica (1). Quanto ao tipo de estudo, encontraram-se pesquisas focadas em: estudos epidemiológicos (1), descritivos (5), transversais (7), longitudinal (1), estudo de caso (1) e revisão de literatura (1). Encontrou-se, também, relato de experiência (1). Como observado, dois artigos abordaram mais de um tipo de estudo.

Em relação aos autores, mencionam-se enfermeiros, antropólogo, educador físico, médico pediatra e nutricionistas. Ressalta-se que a autoria do enfermeiro em todos os artigos era um dos critérios de inclusão estabelecido para este estudo. Dez artigos tinham apenas enfermeiros como autores, dois tinham enfermeiro e nutricionista, um artigo era de autoria de enfermeiro e antropólogo; em apenas um a autoria era de educador físico, enfermeiro e nutricionista e também em apenas um a autoria era de enfermeiro e médico.

Conforme identificado, as publicações analisadas abrangeram temas variáveis, desde o conhecimento, a evolução, o acompanhamento e a qualidade da avaliação nutricional realizada pelo enfermeiro à sua prática no concernente à saúde da criança.

Os estudos selecionados segundo sua abrangência foram agrupados em unidades temáticas, assim denominadas: avaliação nutricional mediante acompanhamento do crescimento e desenvolvimento infantil pelo enfermeiro; fatores determinantes da nutrição da criança e avaliação nutricional como cuidado de enfermagem. Contudo, como alguns artigos tinham maior abrangência, foram incluídos em mais de uma temática.

\section{Avaliação Nutricional mediante Acompanhamento do Crescimento e Desenvolvimento Infantil pelo Enfermeiro}

Com referência a esta temática foram categorizados onze artigos. Todos eles enfocaram a identificação de índices antropométricos, tais como peso, estatura, pregas cutâneas, e perímetros cefálico, torácico e abdominal, como método de avaliação nutricional da criança pelo enfermeiro em suas atividades no contexto hospitalar e/ou na saúde pública, especificamente nas consultas de acompanhamento do crescimento e desenvolvimento da criança.

Neste grupo, as publicações abordaram, na maioria, crianças menores de cinco anos de idade e sadias, enquanto dois artigos eram referentes a crianças desnutridas e crianças menores de um ano de idade portadoras de cardiopatias congênitas, respectivamente.

De acordo com Telles e Barros Filho, ${ }^{7}$ as medidas antropométricas são de fácil obtenção e podem ser consideradas como um método de avaliação nutricional na pediatria. Além das medidas antropométricas, algumas relações entre parâmetros antropométricos foram descritas como formas de avaliação nutricional de crianças logo após o nascimento, objetivando ressaltar a pro-porcionalidade do crescimento intrauterino e pós-natal. 0 índice ponderal (IP), por exemplo, reflete a proporcionalidade do crescimento intrauterino, pois leva em conta peso e comprimento. ${ }^{8}$

Em virtude da limitação de métodos laboratoriais e de composição corpórea, a ava-liação nutricional do recém-nascido continua a se basear em parâmetros antropométricos, como peso, comprimento, perímetros cefálico e braquial e dobras cutâneas. Além dos parâmetros antropométricos, as relações antropométricas são úteis para descrever proporcionalidades corpóreas. ${ }^{9}$

Por meio do acompanhamento do crescimento infantil, com o método de avaliação nutricional se faz a evolução periódica do ganho de peso e é possível avaliar o progresso da criança, identificando aquelas em maior risco de morbimortalidade, prevenindo precocemente a desnutrição. ${ }^{10}$

Rotineiramente, as mães levam seus filhos ao consultório na tentativa de identificar alterações nutricionais 
quando os submetem à antropometria, um dos procedimentos feitos na consulta de puericultura. Nesse sentido, as mães atuam como coadjuvantes no processo de cuidado à criança. Carvalhães e Godoy ${ }^{11}$ consideram o envolvimento da mãe uma característica necessária para que a monitoração do crescimento possa produzir impacto positivo sobre a saúde da criança.

\section{Fatores Determinantes da Nutrição da Criança}

Nove estudos foram agrupados nesta unidade temática cujo objetivo foi identificar os fatores determinantes da nutrição infantil, entre eles, a alimentação e o ambiente. Nos artigos que abordaram temas sobre a alimentação das crianças, estas eram saudáveis e tinham até seis anos de idade.

Segundo Santos e Santana, ${ }^{12}$ a alimentação destina-se a satisfazer as necessidades nutricionais, como também possibilita, em cada ser humano, o desenvolvimento das suas atividades intelectuais, psicológicas, biológicas e culturais em um processo de constante socialização.

Neste aspecto, alguns autores abordaram a dinâmica fisiológica e emocional da criança, por meio da amamentação, considerada uma das primeiras experiências nutricionais do recém-nascido. Embora este receba nutrição intrauterina, atualmente, em decorrência destas experiências, o enfermeiro atua como principal envolvido na questão do aleitamento. ${ }^{13}$

Como observado, as primeiras experiências nutricionais de um indivíduo não guardam relações somente com seu peso quando adulto. Elas também podem interferir na sua suscetibilidade a determinadas doenças crônicas na fase adulta, como obesidade, hipertensão arterial e doenças cardiovasculares. ${ }^{13}$

A alimentação e a nutrição constituem requisitos básicos para a promoção e a proteção da saúde; possibilitam a afirmação plena do potencial de crescimento e desenvolvimento humano, com qualidade de vida e cidadania. ${ }^{14}$ No entanto, alguns estudos relatam as deficiências proteico-energéticas ainda persistentes nas crianças originárias do Norte e Nordeste do Brasil como algo que contraria os pressupostos defendidos pela Política Nacional de Alimentação e Nutrição do país.

Para poder fundamentar adequadamente suas ações de cuidado, na avaliação da condição nutricional da criança, o enfermeiro deve considerar o conhecimento sobre os fatores ambientais, as condições socioeconômicas, os componentes culturais e educativos do meio onde a criança vive.

Segundo evidenciaram estudos realizados em diferentes estados brasileiros, cada ambiente possui suas características singulares, conjunturas específicas e momentos históricos. Estas, possivelmente moduladas por ações sociais, refletem seus próprios resultados de nutrição na heterogeneidade regional da sua nação. ${ }^{15}$

No meio rural, é comum identificar problemas de saúde focalizados nos grupos populacionais mais frágeis, que necessitam de mais atenção e investimento em políticas de saúde. Por esta razão, as crianças jovens inseridas neste contexto são as mais afetadas no relacionado à constante monitoração do crescimento e desenvolvimento. ${ }^{15}$

Para suprir esta deficiência, ações governamentais são instituídas quando se projeta apoio social via creches comunitárias que têm sob sua responsabilidade a oferta alimentar como um cuidado primário, fazendo visível seu importante papel na promoção da saúde e no desenvolvimento integral da criança. ${ }^{12}$

No âmbito hospitalar, a identificação de crianças com agravo nutricional durante o período de internamento propicia adequar o tratamento e estimar o prognóstico, mas a avaliação nutricional deve fazer parte da rotina dos cuidados das crianças. ${ }^{16}$ Neste setor, o papel do enfermeiro está direcionado ao acompanhamento da doença de base da criança, a qual pode demonstrar um agravo suficiente para determinar alterações nutricionais.

\section{A Avaliação Nutricional como um Cuidado de Enfermagem}

Nesta temática, oito publicações foram categorizadas e abordaram a avaliação nutricional como uma prática do cuidado de enfermagem a partir da elaboração de instrumentos de registros da saúde da criança e avaliação nutricional como método diagnóstico.

Em todos os estudos deste grupo, as crianças se encontravam saudáveis. Apenas um artigo não informava a condição de saúde nem a idade da criança. Em dois artigos, as crianças tinham até dois meses de vida, em outros dois, tinham até seis meses e, por último, outro artigo fez alusão a crianças menores de cinco anos de idade.

Um artigo referente a instrumentos de registro da saúde da criança, identificado como revisão bibliográfica, abordava diferentes instrumentos utilizados para avaliação da saúde da criança. A finalidade do estudo, tendo como parâmetro diversos instrumentos, era construir um protocolo de acompanhamento do crescimento e desenvolvimento da criança. ${ }^{17}$ Como observado, a adoção do protocolo visava sistematizar o trabalho nos serviços de saúde, no planejamento de ações e estabelecimento de prioridades que repercutem em melhorias da qualidade da atenção à saúde da população infantil. 
0 instrumento de registro da saúde da criança tem o potencial de propiciar uma base segura para o seguimento da criança e sua família, pois nele o enfermeiro encontra, de forma ordenada, informações sobre antecedentes pré-natais, parto, histórico alimentar e vacinal, estrutura e dinâmica familiares, ambiente físico, relações intrafamiliares e sociais e acompanhamento da criança até o segundo ano de vida. Fundamentado nestes dados, o profissional capacitado poderá interligar as informações disponíveis, guiando a assistência prestada em nível individual e familiar. ${ }^{17}$ Nesse sentido, 0 enfermeiro expressa preocupação constante em realizar um cuidado sistemático, individual e mais qualificado, com vistas à avaliação do seu próprio trabalho.

Com a ampliação dos cuidados de enfermagem junto às famílias, torna-se necessário, em saúde da criança, estruturar a avaliação que envolve a criança, o grupo familiar e o ambiente, buscando abordar o desenvolvimento infantil. ${ }^{17}$ Além disso, motivar mães e/ou cuidadores para uma alimentação adequada e incentivar o acompanhamento do peso pode facilitar a identificação precoce de sinais/sintomas de desnutrição/ sobrepeso.

Conforme mostram estudos que abordam a temática do cuidado de enfermagem nas unidades básicas de saúde, quando $o$ enfermeiro realiza a consulta de enfermagem, ele questiona a mãe quanto aos possíveis fatores que estão interferindo no processo de alimentação da criança, bem como enseja um momento de diálogo e esclarecimento de dúvidas passíveis de influenciar as condições nutricionais do bebê. Entre elas, destaca-se a avaliação das mamas e da sucção da criança durante a amamentação. ${ }^{18}$

A avaliação nutricional como meio de identificar respostas humanas de alteração da nutrição da criança pode ser encontrada em estudos que tratam da investigação da relação peso ao nascer e anemia no decorrer do primeiro ano de vida. Como se sabe, a deficiência de ferro traz consequências deletérias para a saúde dos indivíduos, pois diminui a capacidade física e influi negativamente na capacidade laborativa, tanto individual quanto para a comunidade. Associada ao crescimento, a introdução precoce da dieta de desmame à base de legumes, cereais e leite de vaca pode levar ao desenvolvimento da anemia. ${ }^{19}$

Por esse motivo, mencionada deficiência de nutrientes pode ser minimizada pelo incentivo na continuação do processo de amamentação, uma vez que a presença de vitaminas e enzimas no leite materno facilita a absorção de ferro no organismo infantil. Em face disso, um estudo realizado com mulheres nutrizes apontou que, mesmo reconhecendo a importância do leite materno como ideal para a nutrição e proteção do filho, as mães ainda relatam muitas dificuldades para a manutenção da amamentação, as quais culminam para o desmame precoce e, consequentemente, déficits nutricionais. ${ }^{20}$

À medida que o enfermeiro executa suas tarefas diárias de acompanhamento da saúde da criança em qualquer nível de atenção à saúde, ele tenta identificar respostas humanas aos problemas de saúde reais ou potenciais, caracterizando o diagnóstico de enfermagem mediante a situação clínica de saúde apresentada. A seguir, então, propõe intervenções descritas em atividades de cuidados específicos à criança.

\section{CONSIDERAÇÕES FINAIS}

Foram analisados artigos na íntegra publicados nos últimos sete anos. Verificou-se que a avaliação nutricional infantil tem sido abordada em publicações mais recentes. Como observado, as publicações analisadas abrangeram temas sobre conhecimento, evolução, acompanhamento e qualidade da avaliação nutricional pelo enfermeiro, bem como sua prática concernente à saúde da criança.

Os estudos selecionados segundo sua abrangência foram agrupados em unidades temáticas. Contudo, alguns artigos tinham maior abrangência e foram incluídos em mais de uma temática.

Com a elaboração deste estudo, percebe-se que o enfermeiro tem buscado fundamentação teórica e prática para cuidar da criança com déficit nutricional. Diante disso, conforme a análise temática permitiu identificar, a maior parte dos estudos relaciona a avaliação nutricional à investigação de índices antropométricos como fatores determinantes do crescimento da criança. No entanto, essa atividade ainda está bastante restrita às ações de saúde primárias, desenvolvidas nas unidades básicas de saúde, nas quais são executadas as consultas de puericultura pelo enfermeiro.

Salienta-se, porém, que o número de artigos encontrado não foi representativo diante da quantidade total de artigos publicados nos periódicos selecionados. Este fato revela a necessidade de ampliar a produção científica na área de enfermagem sobre a temática em estudo, porquanto as publicações ainda são bastante restritas ao profissional da nutrição.

Outras lacunas foram destacadas; por exemplo, a maior parte dos estudos enfoca crianças na faixa etária inferior a cinco anos de idade e tem como população crianças sadias. Ademais, algumas situações de saúde/doença que estão fortemente relacionadas com o crescimento e desenvolvimento das crianças, a exemplo de alterações pulmonares, não foram 
abordadas. E apenas um estudo teve como foco criança com cardiopatia congênita. Em face da situação, considera-se importante investir nesta área com vistas a subsidiar o enfermeiro no cuidado e ampliar o corpo de conhecimento teórico da enfermagem acerca da avaliação nutricional da criança em diversas situações de saúde, agudas ou crônicas.

\section{REFERÊNCIAS}

1. Sigulem DM, Devincenzi UM, Lessa AC. Diagnóstico do estado nutricional da criança e do adolescente. J Pediatr 2000; 76(supl 3): 275-83.

2. Ministério da Saúde (BR). Saúde da criança: acompanhamento do crescimento e desenvolvimento infantil. Série cadernos de atenção básica. Brasília (DF); 2002.

3. Conselho Federal de Enfermagem-COFEn. Resolução n 277/2003. Dispõe sobre o regulamento da terapia nutricional [on-line]. [citado 23 abril 2008]. Disponível em: http://www.portalcofen.gov.br/ novoportal.

4.Gil AC. Como elaborar projetos de pesquisa. $4^{\mathrm{a}}$ ed. São Paulo (SP): Atlas; 2002.

5.Leopardi MT. Metodologia da pesquisa na saúde. $2^{\mathrm{a}}$ ed. rev. atual. Florianópolis (SC): UFSC; 2002.

6. Ministério da Saúde (BR). Conselho Nacional de Saúde. Resolução $n^{\circ} 196$, de 10 de outubro de 1996. Diretrizes e normas regulamentadoras de pesquisas envolvendo seres humanos. Inf Epidemiol SUS 1996; 5 (2 supl 3): 13-41.

7.Telles RK, Barros Filho AA. 0 uso da antropometria como método de avaliação da composição corporal em pediatria. Rev Cienc Med 2003; 12(4): 351-63.

8.Cardoso LEB, Falcão MC. Importância da avaliação nutricional de recém-nascidos pré-termo por meio de relações antropométricas. Rev Paul Pediatr 2007; 25(2): 135-41.

9.Brock RS, Falcão MC. Avaliação nutricional do recém-nascido: limitações dos métodos atuais e novas perspectivas. Rev Paul Pediatr 2008; 26(1): 70-6.

10.Carvalho MF, Lira PIC, Romani SAM, Santos IS, Veras AACA, Batista Filho M. Acompanhamento do crescimento em crianças menores de um ano: situação nos serviços de saúde em Pernambuco, Brasil. Cad Saude Publica 2008; 24(3): 675-85.

11.Carvalhaes MABL, Godoy I. As mães sabem avaliar adequadamente o peso das crianças? Rev Nutr 2002; 15(2): 155-62.

12.Santos MB, Santana JSS. Critérios utilizados na alimentação da criança na creche comunitária: um problema de informação? Rev Baiana Saude Publica 2005; 29(2): 160-74.
13.Araújo MFM, Beserra EP, Chaves ES. 0 papel da amamentação ineficaz na gênese da obesidade infantil: um aspecto para a investigação de enfermagem Acta Paul Enferm 2006; 19(4): 450-5.

14. Ministério da Saúde(BR). Secretaria de Atenção à Saúde. Departamento de Atenção Básica. Política nacional de alimentação e nutrição. $2^{a}$ ed. rev. Brasília (DF); 2007.

15. Rocha L, Gerhardt TE, Santos DL. Social heterogeneity and children's nutrition in the rural environment. Rev Latino-am Enfermagem 2007; 15(n esp): 828-36.

16. Péret Filho LA, Penna FGC, Rodrigues FG, Santana DP, Hanan B, Oliveira GNM, et al.. Avaliação nutricional de crianças internadas em enfermaria geral de um hospital público. Pediatr 2005; 27(1): 12-8.

17. Pina JC, Mello DF, Lunardelo SR. Utilização de instrumento de registro de dados da saúde da criança e família e a prática do enfermeiro em atenção básica à saúde. Rev Bras Enferm 2006; 59(3): 270-73.

18. Figueiredo GLA, Mello DF. 0 cuidado de enfermagem às crianças menores de dois anos de idade em uma unidade básica de saúde: utilizando os momentos da vacinação. Acta Paul Enferm 2003; 16(4): 86-90.

\section{NOTAS}

a Trabalho apresentado à disciplina Metodologia Científica do Programa de Pós-Graduação - nível Mestrado, em Enfermagem da Universidade Federal do Ceará (UFC)
Data de recebimento: 05/12/2008 Data de reapresentação: 08/10/2009 Data de aprovação: 05/12/2009 